\title{
Möglichkeiten der Haft- und \\ Untersuchungshaftvermeidung bei \\ jugendlichen und heranwachsenden Straftätern am Beispiel des Kölner Vereins Maßstab e.V.
}

Von Katrin Banike

\begin{abstract}
Haftvermeidungsprojekte für junge Straftäter - ein viel diskutiertes Thema! Doch wie arbeitet eine solche Einrichtung konkret? Was erwartet die jugendlichen und heranwachsenden Täter dort? Welche Ziele werden verfolgt? Diese Thematik soll im folgendem am Beispiel des Kölner Vereins Maßstab e.V. verdeutlicht werden. ${ }^{1}$
\end{abstract}

\section{Allgemeines}

$\int$ er Verein Maßstab e.V. existiert seit 1986 und ist seitdem auf dem Gebiet der Straffälligenhilfe tätig. Seit 1996 betreibt der Verein eine "Jugendwohngemeinschaft für Jugendliche und Heranwachsende zur Vermeidung bzw. Verkürzung von Untersuchungsoder Strafhaft « - so die offizielle Bezeichnung. Es stehen vier Plätze zur Verfügung: Jeder der Bewohner erhält ein eigenes Zimmer, zudem gibt es eine Wohnküche für alle sowie eine große Dachterrasse. Die Zielgruppe sind männliche Jugendliche und Heranwachsende ${ }^{2}$ im Alter von 16-20 Jahren, die meist mehrfach durch das Begehen strafbarer Handlungen auffällig geworden sind. Voraussetzung für eine Aufnahme ist, dass der Bewerber die Mitarbeiter des Maßstabs von seiner Bereitschaft zur Mitwirkung überzeugt hat. Eine Vorauswahl wird aufgrund der hohen Bewerberzahl bereits durch den Sozialdienst, der in der JVA Köln tätig ist, getroffen. Ausgeschlossen ist eine Aufnahme bei Vorliegen einer psychiatrischen Diagnose oder einer im Vordergrund stehenden Drogenproblematik. Auch extrem gewaltbereite Jugendliche und Sexualstraftäter werden nicht aufgenommen. Für solche Fälle ist die Einrichtung nicht ausgelegt. Die durchschnittliche Dauer des Aufenthaltes beträgt 6-15 Monate.
Angebote, Leistungen, Arbeitsmethoden der Einrichtung

Nach dem Konzept des Maßstabs wird der Aufenthalt in der Wohngemeinschaft in drei Phasen unterteilt: Die Aufnahmephase, die Stabilisierungsphase und die Verselbständigungsphase. Die erste Phase kann auch als Orientierungsphase bezeichnet werden, da der neue Bewohner sich nicht nur in der Wohngemeinschaft einleben soll, sondern auch seine berufliche und private Zukunft mit ihm zusammen geplant wird. In dieser Phase wird von Seiten der Betreuer versucht eine Vertrauensbasis zu schaffen. Hier ist es hilfreich, dass es sowohl eine weibliche Betreuerin als auch einen männlichen Betreuer gibt, da manche der jungen Straftäter eher zu einer Frau und manche eher zu einem Mann als Bezugsperson tendieren. In dieser Anfangsphase läuft nach Aussage der Betreuer viel über Intuition und wenig über klassische pädagogische Methoden. Hinsichtlich der beruflichen Planung geht es vorrangig darum, ob die Schulbildung fortgeführt werden soll bzw. welche Ausbildung oder Arbeitsstelle angestrebt wird. In der zweiten Phase sollen die gefundenen Ziele realisiert werden bzw. im Falle der Nichtrealisierung den vorhandenen Möglichkeiten angepasst werden. Der Verein Maßstab e.V. bietet in den vereinseigenen Zweckbetrieben Schreinerei,
Innenausbau und Restaurant Praktika und Lehrstellen an. Man kann hier als Argument entgegenhalten, dass es den jungen Straftätern zu leicht gemacht wird, wenn ihnen von Seiten des Vereins auch noch eine Arbeitsbzw. Ausbildungsstelle vermittelt wird. Hiergegen lässt sich anführen, dass der junge Straftäter auf dem heutigen Arbeitsmarkt schlechte bis keine Chancen hat, überhaupt etwas zu finden, um auf legalem Wege sein Geld zu verdienen. Diese Tatsache kann zu einer weiteren Schwächung seines meist angeschlagenen Selbstbewusstseins führen. Wenn ihm jedoch der Start in das Berufsleben erleichtert wird, so führt das zu einem positiven Erlebnis und mehr Selbstbewusstsein. Die Schwierigkeiten hinsichtlich einer Arbeitsstelle liegen meist darin, dass es den Jugendlichen schwer fällt, einer regelmäßigen Arbeit nachzugehen. Hier ist Handlungsbedarf gegeben: Die Betreuer müssen die Bewohner zum Durchhalten motivieren, Rückschläge auffangen und auch evtl. auftretende Probleme am Arbeitsplatz lösen. In der dritten und letzten Phase steht die Zeit nach der Entlassung im Mittelpunkt: Der Jugendliche soll selbständiger werden, auch in privater Hinsicht. Hierzu gehört, dass der Klient den Umgang mit Geld lernt, sich Freizeitaktivitäten außerhalb des Vereins sucht. Kurz vor seiner Entlassung wird eine passende Wohnung ge- sucht. Zudem wird eine Nachbetreuung angeboten.

Hauptansatzpunkt der Arbeit mit den jungen Straftätern ist das sog. »lebenspraktische Training «. Sie lernen eine Struktur in ihren Tagesablauf hineinzubringen: Frühes Aufstehen, sich gewissen gesellschaftlichen Rahmenbedingungen anzupassen, selbständiges Einkaufen und Kochen, Ordnung halten etc.. Dies beinhaltet aber auch die Suche nach einem geeigneten Job, u.a. werden Bewerbungstrainings durchgeführt. Zu den Methoden des Vereins zählt zudem die Einhaltung von Regeln, um den Jugendlichen Grenzen aufzuzeigen. Jeder Neuzugang hat daher mit der Einrichtung einen Vertrag zu unterschreiben. Damit verpflichtet er sich, keine illegalen Drogen oder Alkohol zu konsumieren; sich auf Verlangen der Betreuer einem Drogentest $\mathrm{zu}$ unterziehen sowie dem Verzicht auf Waffen, Gewalt und das Begehen von Straftaten. Es besteht eine Teilnahmenpflicht an gemeinsamen Aktivitäten, der Putz- und Dienstplan muss eingehalten werden. Des weiteren muss ein Tag- und Nacht-Rhythmus, der sich an den gesellschaftlich üblichen Werk- und Freizeiten orientiert, auch dann eingehalten werden, wenn der Jugendliche sich nicht in einem Beschäftigungsverhältnis befindet. Bei Verstößen wird auf ein strukturiertes Sanktionssystem zurückgegriffen. Die- 
se Regeln dienen nicht nur dazu, gewisse Grenzen zu setzen, sondern sollen das »lebenspraktische Training « unterstützen. Dieses wiederum soll den Jugendlichen helfen vor allem im Berufsleben zurechtzukommen, denn häufig scheitert eine angefangene Ausbildung daran, dass der Jugendliche regelmäßig zu spät kommt oder sich den dortigen Bedingungen nicht anpassen kann. Eine 24-Stunden-Bereitschaft seitens der Betreuer ist nicht gegeben, diese sind im Notfall aber über Handy zu erreichen.

Die Ziele des Vereins sind neben der Lebensführung ohne Straftaten, die Entwicklung größtmöglicher Selbstbestimmung und die Entwicklung sozialer Verantwortung.

Der Hauptunterschied zum Strafvollzug ist, dass die Jugendlichen das neu erlernte Wissen und Können direkt im Alltagsleben ausprobieren können. Erfolgserlebnisse wirken dabei als positive Verstärker und neu erworbene Verhaltensmuster manifestieren sich. Die erlernten Verhaltensmuster führen dazu, dass sie sich besser in das Gesellschaftsleben integrieren können. Rückfälle in alte Verhaltensmuster werden von den Betreuern erkannt und mit den Betroffenen in Einzelgesprächen thematisiert. In einigen Jugendstrafanstalten werden zwar auch Trainingsprogramme unterschiedlichster Art angeboten z.B. Anti-Aggressivitätstraining. Aber hier ist der Weg des Trainings bis zur konkreten Alltagssituation zu lang: Das Erlernte hat sich nicht genügend verfestigt, um sofort im Alltagsleben zu funktionieren. Dies führt nach der Entlassung nicht nur häufig zu Frustration und den Rückfall in alte Verhaltensmuster, sondern auch dazu, das möglicherweise eine erneute Straftat begangen wird.

Das Konzept der Jugendwohngemeinschaft geht jedoch nur auf, wenn der Jugendliche vor, während oder spätestens nach seinem Aufenthalt dort, erkennt, dass er sich selbst und seine Verhaltensweisen ändern muss, um in Zukunft straffrei zu bleiben. Fehlt ihm diese Einsichtsfähigkeit, so wird er den Kreislauf der kriminellen Karriere nicht durchbrechen können. Diese Erfahrung musste auch der Verein Maßstab e.V. machen. Langzeitstudien gibt es bisher nicht, während des Aufenthaltes blieben jedoch $80 \%$ der Jugendlichen straffrei. Diese Quote ergibt sich aus der
Evaluationsliste der Jugendwohngemeinschaft, die seit 1999 geführt wird. Der Zeitraum dieser Liste erstreckt sich jeweils über ein Kalenderjahr. In dieser Liste werden neben den Daten der Jugendlichen folgende Spalten für jeden einzelnen Bewohner geführt und bei Vorliegen entsprechend eingetragen: Verweildauer in Monaten insgesamt/reguläre Beendigung/JA stellt ein/Abbruch wg. Regelverstoß/Abbruch wg. erneuter Straffälligkeit während des Aufenthaltes/Abbruch auf Wunsch des Bewohners/Abbruch aus anderen Gründen/straftatenfreies Leben während des Aufenthaltes/erneute Straffälligkeit innerhalb von 6 Monaten nach Entlassung/erneute Straffälligkeit innerhalb von 12 Monaten nach Entlassung/Bewohner hat schulische und berufliche Orientierung gewonnen/Bewohner ist ein Ausbildungsverhältnis eingegangen/Abbruch eines Ausbildungsverhältnisses nach 1-3 Monaten/Abbruch eines Ausbildungsverhältnisses nach mehr als 3 Monaten sowie Ausbildungsverhältnis bestand bei Beendigung fort.

Nicht nur die im Gegensatz zu den Statistiken der Jugendstrafanstalten positive Bilanz ist ein Argument für das Projekt, sondern auch die Kosten: Die Kosten der Unterbringung betragen $120 €$ pro Tag und Person. Die Unterbringung eines Jugendlichen im Jugendstrafvollzug bzw. in der Untersuchungshaft liegen ca. 20-30 € über diesem Tagessatz. Die Jugendlichen, die während der Unterbringung in der Wohngemeinschaft erwerbstätig sind, haben an die Kostenträger einen Eigenanteil von bis zu 70\% zu leisten. Durch das Projekt Haftvermeidung ergeben sich jährlich hohe Einsparungen für das Land NRW.

Da in einem solchen Projekt die negativen Auswirkungen der traditionellen Jugendstrafe vermieden werden, ohne dass sich die Rückfallquote erhöht, ist diesem unbedingter Vorzug zu geben.

Die Verfasserinn ist Sozialarbeiterin in Köln

\section{Fußnoten:}

1 Die Ausführungen betreffen nur den Verein Maßstab e.V., da nur dieser von der Verfasserin ausführlich untersucht worden ist und keine anderen Einrichtungen ähnlicher Art.

$2 \mathrm{Im}$ folgenden Text wird aufgrund der besseren Lesbarkeit nur noch von dem Jugendlichen gesprochen.

TERMINAL

\title{
Europäisches Parlament wehrt sich gegen Fluggastdaten-Transfer an US-Sicherheitsbehörden
}

\author{
Von Rolf Gössner
}

Das Europäische Parlament wird den Europäischen Gerichtshof in Luxemburg anrufen, um die Annullierung eines Abkommens zu erreichen, das EU-Rat und -Kommission im Mai 2004 gegen das ausdrückliche Votum des Parlaments und der EUDatenschutzbeauftragten mit den USA geschlossen hatten. In dem Abkommen geht es um die Übermittlung von Flugpassagierdaten an Sicherheitsbehörden der Vereinigten Staaten.

Von dem Datentransfer sind jährlich über zehn Millionen Flugpassagiere aus Europa, die in die USA fliegen oder über die USA weiterreisen, unmittelbar betroffen. Schon bevor sie auch nur einen Fuß auf den Boden des Landes gesetzt haben, sind die US-Sicherheitsbehörden über sie umfassend informiert, haben ihre Schlüsse aus den zum Teil hochsensiblen Informationen gezogen, Verdachtsmomente ventiliert oder Verdächtigungen konstruiert. Die gelieferten Daten können nach allen Richtungen verarbeitet, durchgerastert und mit einer Unzahl anderer polizeilicher, geheimdienstlicher oder auch privater Dateien abgeglichen werden, um Bewegungsbilder und Persönlichkeitsprofile $\mathrm{zu}$ erstellen und Verdächtige herauszufiltern.

Auch unbescholtene Fluggäste müssen verstärkt damit rechnen, dass sie auf diese Weise zu Opfern rigider Antiterror-Maßnahmen werden und sich wie Verbrecher behandeln lassen müssen. Die Datenübermittlung kann letztlich zu peinlichen Verhören und erkennungsdienstlicher Behandlung führen, $\mathrm{zu}$ willkürlichen Festnahmen und Inhaftierungen und schließlich zu Ausweisungen auch vollkommen unschuldiger Personen. Beispiele hierfür gibt es schon genug. Begründet wird all dies mit der »Bekämpfung des Terrorismus sowie sonstiger Verbrechen transnationaler Art, einschließlich der organisierten Kriminalität«.

Doch die große Mehrheit der Europa-Parlamentarier vermisst einen ausreichenden Schutz für die per Online-Zugriff übermittelten Daten. Die erzwungene Datenübermittlung verstoße gegen Grundrechte und völkerrechtlich garantierte Prinzipien des europäischen Datenschutzes, zumal die Daten auch an Behörden anderer Staaten weitergegeben werden dürfen. Datenschützer halten den Datentransfer, der Fluggäste aus der EU zu gläsernen Passagieren macht, für eine unverhältnismäßige und ungeeignete Maßnahme, die weit über das Ziel der Terrorbekämpfung hinausschieße und sich auch mit dem anerkennenswerten Sicherheitsinteresse nicht rechtfertigen lasse.

Dr. Rolf Gössner, Rechtsanwalt und Publizist, ist Präsident der "Internationalen Liga für Menschenrechte", die eine gerichtliche Überprüfung des DatentransferAbkommens angemahnt hat. 